\title{
Adaptive Turbo Trellis Coded Modulation Aided Distributed Space-Time Trellis Coding for Cooperative Communications
}

\author{
Soon Xin Ng, Chuyi Qian, Dandan Liang and Lajos Hanzo \\ School of Electronics and Computer Science, University of Southampton, SO17 1BJ, United Kingdom. \\ Tel: +44-23-8059 3125, Fax: +44-23-8059 4508 \\ Email: $\{$ sxn,cq1g08,dl4e08,lh\}@ecs.soton.ac.uk
}

\begin{abstract}
In this contribution, we propose an adaptive Turbo Trellis Coded Modulation (TTCM) aided Distributed Space-Time Trellis Coding (STTC) scheme for cooperative communication over quasi-static Rayleigh fading channels. An adaptive TTCM (ATTCM) scheme is employed by the source node during the first transmission period for reliably conveying the source bits to $N$ number of relay nodes by appropriately adjusting the coderate and modulation mode according to the near-instantaneous channel condition. The TTCM switching thresholds are chosen to ensure that the Bit Error Ratio (BER) at each relay node becomes lower than $10^{-6}$ in order to minimise the potential error propagation imposed by the relay nodes. During the second transmission period, an $N$-antenna assisted Distributed STTC (DSTTC) scheme is created with the aid of the aforementioned $N$ single-antenna relay nodes. More specifically, the $N$ relay nodes are utilised to form STTC codewords based on the re-encoded TTCM symbols of the relays for transmission to the destination node. At the destination node, iterative extrinsic information exchange is performed between the STTC and TTCM decoders for recovering the original source bits. It is shown that the proposed ATTCM-DSTTC scheme requires $12 \mathrm{dBs}$ less transmission power in comparison to a standard TTCM scheme when aiming for a frame error ratio of $10^{-3}$.
\end{abstract}

Index Terms-Cooperative Diversity, Turbo Trellis Coded Modulation, Distributed Space-Time Trellis Codes, Adaptive Coded Modulation.

\section{INTRODUCTION}

The wireless communication systems of future generations are required to provide reliable transmissions at high data rates in order to offer a variety of multimedia services. Space time coding schemes such as Space-Time Trellis Coding (STTC) [1] employ multiple transmitters and receivers. They are among the most efficient techniques designed for providing a high diversity gain, especially for slowly-fading quasi-static Rayleigh fading channels, where the channel's envelope remains near-constant within a transmission frame albeit varies from frame to frame. Hence, all symbols of a transmission frame tend to fade together. However, when using multiple antennas at the mobile unit it is difficult to eliminate the correlation of the signals due to its limited size. In order to circumvent this problem, cooperative diversity schemes were proposed in [2]-[5]. More specifically, each

The financial support of the EPSRC UK and that of the European Union under the auspices of the Optimix project is gratefully acknowledged. mobile unit collaborates with a few partners for the sake of reliably transmitting its own information and that of its partners jointly, which emulates a virtual Multiple-Input MultipleOutput (MIMO) scheme. The two most popular collaborative protocols used between the source, relay and destination nodes are the Decode-And-Forward (DAF) as well as the AmplifyAnd-Forward (AAF) schemes.

Although a strong channel code can be used for mitigating the potential error propagation in the DAF scheme when communicating over uncorrelated Rayleigh fading channels, its performance becomes limited when communicating over quasi-static Rayleigh fading channels, due to the lack of temporal diversity within a transmission frame. In order to counteract the time-varying nature of the mobile radio channels, near-instantaneous adaptive coded modulation schemes [6]-[8] have been proposed, where a higher-rate code and/or a higherorder modulation mode are employed, when the instantaneous estimated channel quality is high in order to increase the number of Bits Per Symbol (BPS) transmitted. Conversely, a more robust lower-rate code and/or a lower-order modulation mode are employed, when the instantaneous channel quality is low, in order to improve the mean Bit Error Ratio (BER) performance.

When communicating over quasi-static Rayleigh fading channels, each transmission frame effectively experiences an Additive White Gaussian Noise (AWGN) channel with a received SNR determined by the constant fading coefficient and the noise power. Turbo Trellis Coded Modulation (TTCM) [9], which employs two identical parallel-concatenated Trellis Coded Modulation (TCM) [10] schemes as component codes, is one of the most powerful channel coding scheme designed for communicating over AWGN channels. In this contribution, we will employ Adaptive TTCM (ATTCM) for protecting the source-to-relay links, where the effective throughput range is given by $\eta=\{0,1,2,3,5\}$ BPS. A virtual MIMO system in the form of a Distributed STTC (DSTTC) scheme will be created by the cooperating relay nodes in order to circumvent the quasi-static nature of Rayleigh fading channels between the relay nodes and the destination node. In our study, both the source node and the relay nodes are equipped with a single transmit antenna, while the destination node, which could be a base-station, is equipped with two received antennas. 
This paper proposed an effective solution for mitigating the lack of temporal diversity, when communicating over quasistatic Rayleigh fading channels. On one hand, the ATTCM scheme effectively realised the full-potential of various TTCM schemes, when communicating over the source-to-relay links, where error propagation imposed by the DAF-aided relay nodes is minimised. On the other hand, the DSTTC scheme offers spatial diversity to the relay-to-destination links for assisting the STTC-TTCM decoder at the destination node to minimise the probability of decoding errors.

The paper is organised as follows. Our system model is described in Section II, while our system design is outlined in Section III. Our results are discussed in Section IV, while our conclusions are offered in Section V.

\section{SYSTEM MOdEL}

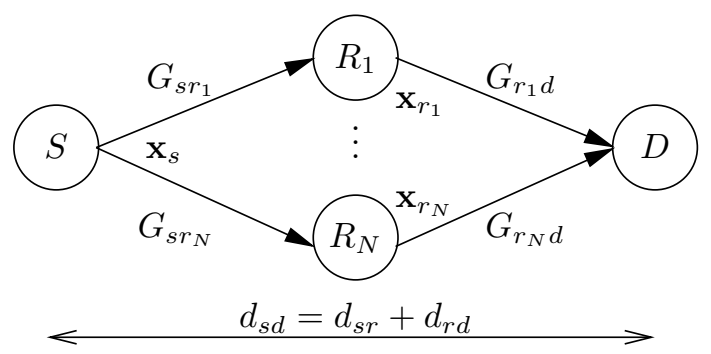

Fig. 1. Schematic of a two-hop relay-aided system, where $d_{a b}$ is the geographical distance between node $a$ and node $b$.

The schematic of a two-hop relay-aided system is shown in Fig. 1, where the source node $(s)$ transmits a frame of coded symbols $\mathbf{x}_{s}$ to $N$ number of relay nodes $(r)$ during the first transmission period. Each relay node first decodes and re-encodes the information. Then all the $N$ cooperating relay nodes will collectively form a virtual MIMO frame of coded symbols $\mathbf{X}_{r}=\left[\mathbf{x}_{1}, \mathbf{x}_{2}, \ldots, \mathbf{x}_{N}\right]^{T}$ for transmission to the destination node during the second transmission period. The communication links seen in Fig. 1 are subject to both free-space propagation path loss as well as to quasi-static Rayleigh fading. We consider $N=2$ relay nodes in this paper. Each source and relay node is equipped with a single antenna, while the destination node is considered to be a base-station assisted by $M=2$ receive antennas.

Let $d_{a b}$ denote the geometrical distance between nodes $a$ and $b$. The path loss between these nodes can be modelled by [11]:

$$
P(a b)=K / d_{a b}^{\alpha},
$$

where $K$ is a constant that depends on the environment and $\alpha$ is the path-loss exponent. For a free-space path loss model we have $\alpha=2$. The relationship between the energy $E_{s r_{i}}$ received at the $i$ th relay node and that of the destination node $E_{s d}$ can be expressed as:

$$
E_{s r_{i}}=\frac{P\left(s r_{i}\right)}{P(s d)} E_{s d}=G_{s r_{i}} E_{s d},
$$

where $G_{s r_{i}}$ is the geometrical-gain [11] experienced by the link between the source node and the $i$ th relay node with respect to the source-to-destination link as a benefit of its reduced distance and path loss, which can be computed as:

$$
G_{s r_{i}}=\left(\frac{d_{s d}}{d_{s r_{i}}}\right)^{2}
$$

Similarly, the geometrical-gain of the $i$ th relay-to-destination link with respect to the source-to-destination link can be formulated as:

$$
G_{r_{i} d}=\left(\frac{d_{s d}}{d_{r_{i} d}}\right)^{2}
$$

Naturally, the geometrical-gain of the source-to-destination link with respect to itself is unity, i.e. we have $G_{s d}=1$.

The $k$ th received signal at the $i$ th relay node during the first transmission period, where $N_{s}$ symbols are transmitted from the source node, can be written as:

$$
y_{s r_{i}, k}=\sqrt{G_{s r_{i}}} h_{s r_{i}} x_{s, k}+n_{r_{i}, k},
$$

where $k \in\left\{1, \ldots, N_{s}\right\}, i \in\{1, \ldots, N\}$ and $h_{s r_{i}}$ is the quasistatic Rayleigh fading coefficient between the source node and the $i$ th relay node, while $n_{r_{i}, k}$ is the AWGN having a variance of $N_{0} / 2$ per dimension. The $j$ th symbol received at the destination node during the second transmission period, where $N_{r}$ symbols are transmitted from each relay node, is given by:

$$
y_{r d, j}=\sum_{i=1}^{N} \sqrt{G_{r_{i} d}} h_{r_{i} d} x_{r_{i}, j}+n_{d, j},
$$

where $j \in\left\{1, \ldots, N_{r}\right\}$ and $h_{r_{i} d}$ denotes the quasi-static Rayleigh fading coefficient between the $i$ th relay node and the destination node, while $n_{d, j}$ is the AWGN having a variance of $N_{0} / 2$ per dimension. Note that the power transmitted by each relay node is normalised to ensure that $\sum_{i=1}^{N}\left|x_{r_{i}, j}\right|^{2}=1$.

If $x_{a, j}$ is the $j$ th symbol transmitted from node $a$ equipped with a single transmit antenna, the average received Signal to Noise power Ratio (SNR) experienced at each receive antenna at node $b$ is given by:

$$
\mathrm{SNR}_{r}=\frac{\mathrm{E}\left\{G_{a b}\right\} \mathrm{E}\left\{\left|h_{a b}\right|^{2}\right\} \mathrm{E}\left\{\left|x_{a, j}\right|^{2}\right\}}{N_{0}}=\frac{G_{a b}}{N_{0}},
$$

where $\mathrm{E}\left\{\left|h_{a b}\right|^{2}\right\}=1$ and $\mathrm{E}\left\{\left|x_{a, j}\right|^{2}\right\}=1$. For the ease of analysis, we define the ratio of the power transmitted from node $a$ to the noise power encountered at the receiver of node $b$ as:

$$
\mathrm{SNR}_{t}=\frac{\mathrm{E}\left\{\left|x_{a, j}\right|^{2}\right\}}{N_{0}}=\frac{1}{N_{0}} .
$$

Hence, we have:

$$
\begin{aligned}
\mathrm{SNR}_{r} & =\mathrm{SNR}_{t} G_{a b} \\
\gamma_{r} & =\gamma_{t}+10 \log _{10}\left(G_{a b}\right)[\mathrm{dB}]
\end{aligned}
$$

where $\gamma_{r}=10 \log _{10}\left(\mathrm{SNR}_{r}\right)$ and $\gamma_{t}=10 \log _{10}\left(\mathrm{SNR}_{t}\right)$. Therefore, we can achieve the desired $\mathrm{SNR}_{r}$ either by changing the transmit power or by selecting a relay node at a different geographical location. 


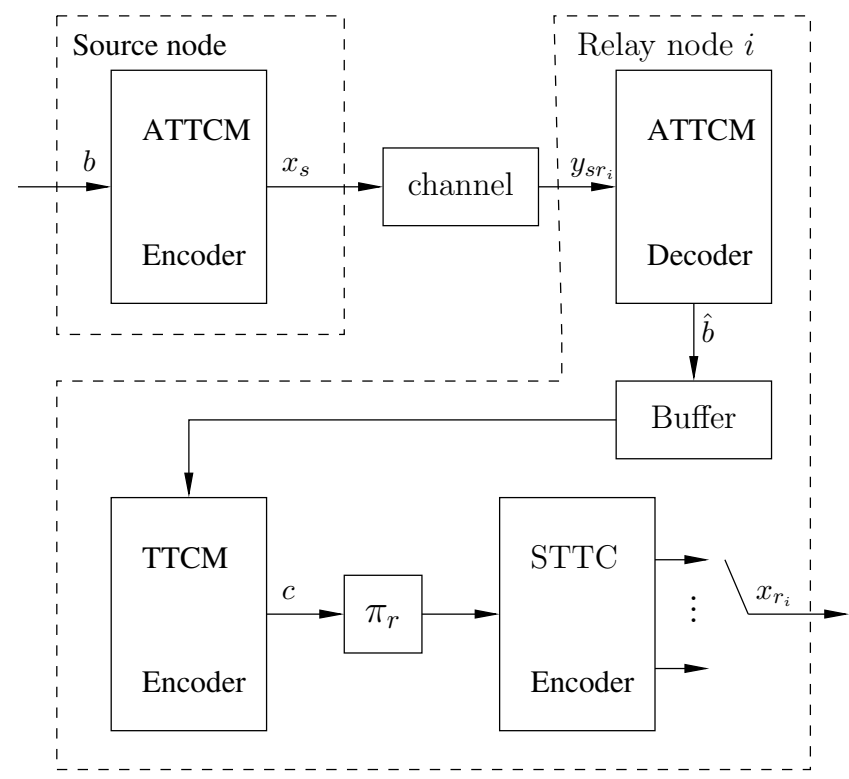

Fig. 2. The schematic of the proposed ATTCM-DSTTC system.

\section{System Design}

If each relay node is equipped with a single antenna, a non-adaptive scheme would require a high transmit power in order to maintain a low number of decoding errors at the relay node, even when a powerful channel encoder is utilised for communicating over quasi-static Rayleigh fading channels. This is due to the lack of temporal diversity within a transmission frame. Hence, if the destination node is equipped with two antennas, a non-cooperative coded scheme may potentially outperform the DAF-aided cooperative coded schemes assisted by single-antenna relay nodes due to the high error inflicted by each relay node, when communicating over quasi-static Rayleigh fading channels. Furthermore, unless a separate power-control loop is provided for the source-to-relay links, the associated BER would be high. Finally, the cochannel interference also exhibits a substantial fluctuation. Due to these reasons, we proposed a near-instantaneous adaptive coded modulation scheme for protecting the source-to-relay links.

In our ATTCM-DSTTC scheme, we consider the following five TTCM modes:

1) No transmission (NoTx): 0 BPS,

2) TTCM-4PSK: 1 BPS,

3) TTCM-8PSK: 2 BPS,

4) TTCM-16QAM: 3 BPS,

5) TTCM-64QAM: 5 BPS,

where all TTCM schemes employ memory-three TCM component codes [12]. We consider $N=2$ relay nodes in the system. The signal received at each relay node is decoded according to the TTCM mode used and the decoded bits are stored in a buffer, as shown in Fig. 2. Then, a fixed-mode TTCM scheme, namely TTCM-4PSK, is used at each relay node in order to re-encode the decoded bits stored in the buffer. The re-encoded TTCM symbols are then symbol-interleaved using $\pi_{r}$ and passed to an STTC encoder. Since each relay node has a single antenna, the first relay node will act as the first antenna of a two-antenna STTC scheme, while the second relay node will act as the the corresponding second antenna. Our destination node is a base-station equipped with $M=2$ receive antennas. Hence, a virtual $(2 \times 2)$-element MIMO system is created between the relay and destination nodes. Finally, iterative

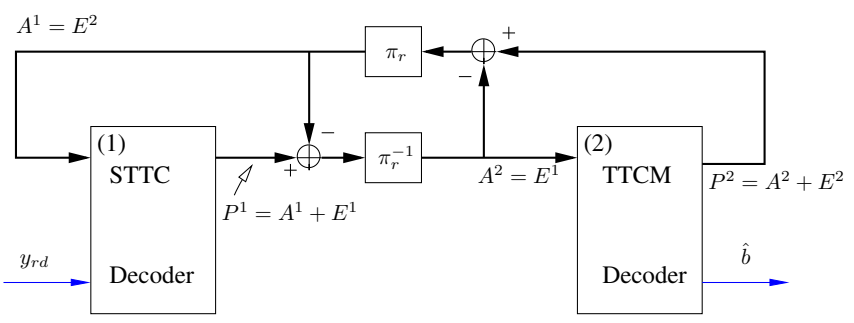

Fig. 3. The schematic of the STTC-TTCM decoder.

decoding between the STTC and TTCM decoders is carried out at the destination node. The schematic of the STTC-TTCM decoder is shown in Fig. 3, where extrinsic information is passed between the two decoders in the form of probability vectors. More specifically, each of the two constituent decoders is labelled with a round-bracketed index. The notations $P$, $E$ and $A$ denote the a posteriori, extrinsic and a priori symbol probabilities, respectively. The probabilities associated with one of the two constituent decoders are differentiated by the superscripts of 1 and 2. The notations $\pi_{r}$ and $\pi_{r}^{-1}$ denote the symbol-based interleaver and deinterleaver, respectively.

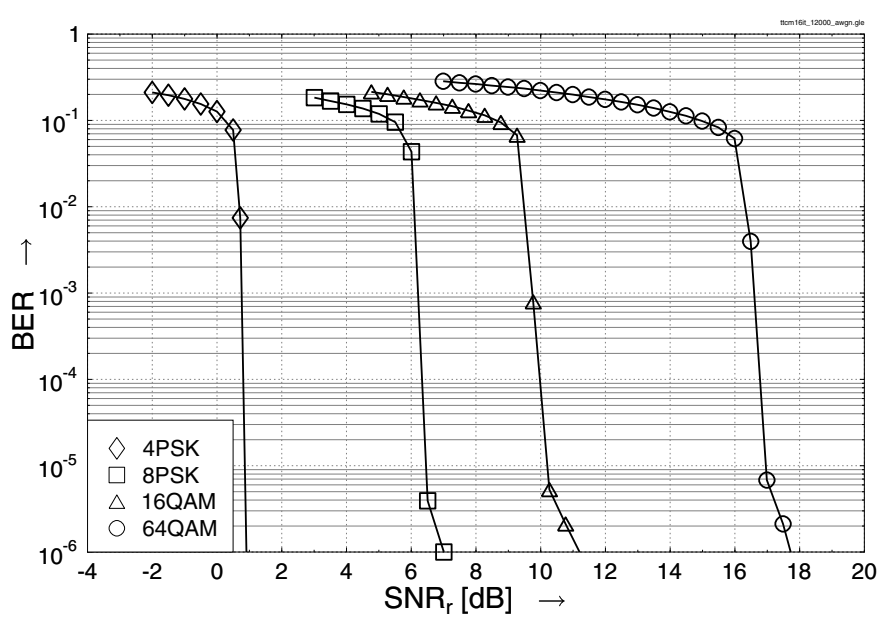

Fig. 4. BER versus $\mathrm{SNR}_{r}$ performance of the various TTCM modes using a frame length of $N_{s}=12000$ symbols when communicating over AWGN channels. 16 turbo iterations is invoked in each TTCM decoder.

The ATTCM switching thresholds $\Gamma=\left[\gamma_{0}, \gamma_{1}, \gamma_{2}, \gamma_{3}\right]$ are determined based on the performance of each of the four TTCM modes in the AWGN channel shown in Fig. 4, where we have $\mathrm{SNR}_{r}=\mathrm{SNR}_{t}$ when $G_{s r_{i}}=1$. Specifically, the ATTCM mode switching operation is based on the following algorithm:

$$
\text { Mode }= \begin{cases}\text { NoTx, } & \text { if } \gamma_{r} \leq \gamma_{0} \\ \text { TTCM-4PSK, } & \text { if } \gamma_{0}<\gamma_{r} \leq \gamma_{1} \\ \text { TTCM-8PSK, } & \text { if } \gamma_{1}<\gamma_{r} \leq \gamma_{2} \\ \text { TTCM-16QAM, } & \text { if } \gamma_{2}<\gamma_{r} \leq \gamma_{3} \\ \text { TTCM-64QAM, } & \text { if } \gamma_{3}<\gamma_{r}\end{cases}
$$


where $\gamma_{r}=\sqrt{G_{s r_{i}}}\left|h_{s r_{i}}\right|^{2} / N_{0}$ is the instantaneous received SNR at a given relay node. In order to minimise the potential error propagation imposed by the relay nodes, we chose the switching thresholds to ensure that the BER at the relay node is lower than $10^{-6}$, which is given by $\Gamma=[1.5,8.0,12.0,18.5] \mathrm{dB}$. Since we have $N=2$ source-torelay links, the ATTCM mode switching is based on the link having the lowest instantaneous received SNR.

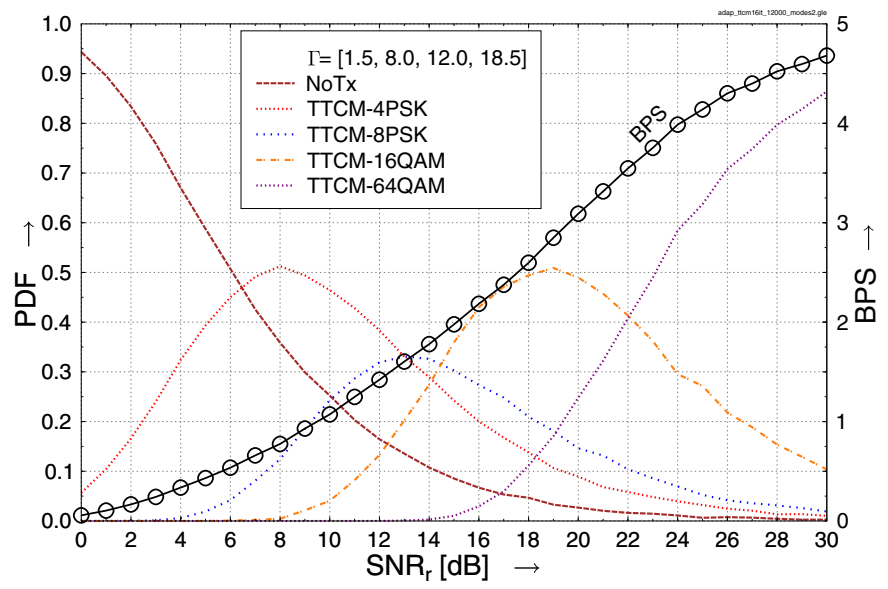

Fig. 5. The PDF of each TTCM mode chosen for a given $\mathrm{SNR}_{r}$ as well as the corresponding BPS values, when communicating over quasi-static Rayleigh fading channels and maintaining a BER below $10^{-6}$.

The Probability Density Function (PDF) of each TTCM mode chosen for a given $\mathrm{SNR}_{r}$ is shown in Fig. 5 together with the corresponding BPS curve. Note from Fig. 5 that as $\mathrm{SNR}_{r}$ increases, the higher-order TTCM modes are chosen more frequently compared to the lower-order counterparts. As a result, the BPS throughput increases smoothly, as $\mathrm{SNR}_{r}$ increases.

\section{RESULTS AND DiscUSSIONS}

In order to benchmark our ATTCM-DSTTC scheme, we have also studied the following five non-cooperative schemes: uncoded BPSK-1x2, BPSK-STTC-2x2, TTCM-4PSK-1x1, TTCM-4PSK-1x2 and TTCM-4PSK-STTC-2x2, where the notation $N \times M$ denotes a system employing $N$ transmitters ${ }^{1}$ and $M$ receivers. All destination nodes in the benchmark schemes employ two receivers. The Frame Error Ratio (FER) performance of these four non-cooperative schemes is plotted together with that of the proposed ATTCM-DSTTC-2x2 scheme in Fig. 6.

The $N=2$ relay nodes are located at the mid-point between the source and destination nodes, hence we have $G_{s r_{i}}=G_{r_{i} d}=4$, which corresponds to $6 \mathrm{dBs}$ geometricalgain for both the source-to-relay and relay-to-destination links. As seen from Fig. 6, the uncoded BPSK-1x2 scheme requires $\mathrm{SNR}_{t}=23 \mathrm{dBs}$ in order to achieve an FER of $10^{-3}$. With the aid of two transmit antennas, the BPSK-STTC-2x2 scheme requires $7 \mathrm{dBs}$ less transmit power to achieve the same FER of

${ }^{1}$ Or $N$ number of relay nodes as in the case of our proposed ATTCMDSTTC scheme.

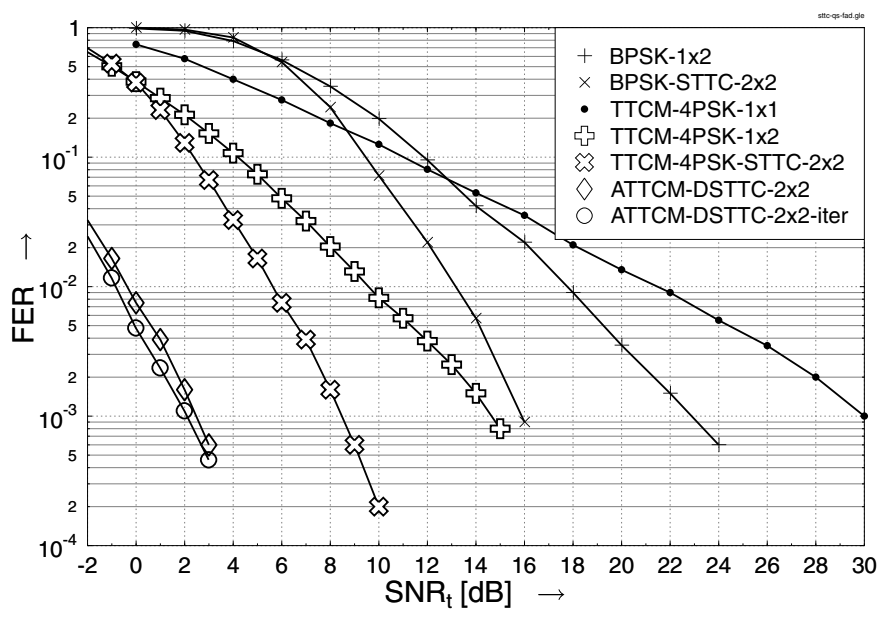

Fig. 6. FER versus $\mathrm{SNR}_{t}$ performance of the BPSK, BPSK-STTC, TTCM4PSK, TTCM-4PSK-STTC and ATTCM-DSTTC schemes when communicating over quasi-static Rayleigh fading channels. The notation $N \times M$ denotes a system employing $N$ transmitters and $M$ receivers. A BER below $10^{-6}$ is maintained at the relay nodes of the ATTCM-DSTTC scheme.

$10^{-3}$, as compared to the single transmit antenna aided BPSK$1 \times 2$ scheme. When the TTCM-4PSK scheme is introduced to replace the BPSK scheme, more than $6 \mathrm{dBs}$ gain can be achieved for both the single-antenna and twin-antenna aided systems. As a benefit of the space-diversity offered by the STTC component and the temporal diversity offered by the TTCM component, the TTCM-4PSK-STTC- $2 \times 2$ scheme outperforms the BPSK-1x2 scheme by more than $14 \mathrm{dBs}$ at FER $=10^{-3}$. Further $6 \mathrm{dBs}$ of geometrical-gain was achieved by the proposed ATTCM-DSTTC-2x2 scheme. Observe in Fig. 6 that the cooperative ATTCM-DSTTC-2x2 scheme outperforms the non-cooperative TTCM-4PSK-1x2 with the aid of two relay nodes, by approximately $12 \mathrm{dBs}$, when aiming for a FER of $10^{-3}$. The above-mentioned BPSK-STTC-2x2, TTCM-4PSK-STTC-2x2, ATTCM-DSTTC-2x2 schemes do not perform decoding iterations between the inner STTC decoder and the corresponding outer decoder. A further coding gain of $0.5 \mathrm{~dB}$ is attained by the ATTCM-DSTTC-2x2-iter scheme with respect to the ATTCM-DSTTC- $2 \times 2$ scheme, when a single iteration is invoked between the STTC and TTCM decoders.

Notice from Fig. 6 that the TTCM-4PSK-1x1 scheme requires $\mathrm{SNR}_{t}=30 \mathrm{dBs}$ in order to achieve an FER of $10^{-3}$. Hence, if a non-adaptive TTCM-4PSK scheme is employed at the single-antenna aided source node and all the single-antenna assisted relay nodes are located at the mid-point between the source and destination nodes, then $\mathrm{SNR}_{t}=30-6=$ $24 \mathrm{dBs}$ is required between each of the source-to-relay links. On the other hand, the non-cooperative TTCM-4PSK-1x2 scheme requires only $\mathrm{SNR}_{t} \approx 14.5 \mathrm{dBs}$ to achieve the same FER, when the destination node is equipped with two receive antennas. Hence, when the destination node is assisted by two receive antennas, a DAF-aided cooperative scheme employing a fix-mode coded scheme at the source node would not be able to outperform the non-cooperative coded scheme due to the high error inflicted by each single-antenna assisted relay node for transmission over quasi-static Rayleigh fading channels. 
This observation further justifies the rationale of employing adaptive coded modulation at the source node.

It is also worth mentioning that at $\mathrm{SNR}_{t}=3 \mathrm{~dB}$ as shown in Fig. 6, the corresponding average received SNR at each relay node is given by $\mathrm{SNR}_{r}=3+10 \log _{10}(4)=9 \mathrm{dBs}$, when the relay nodes are located at the mid-point between the source and destination nodes. This $\mathrm{SNR}_{r}$ value corresponds to $\mathrm{BPS} \approx 1.0$ according to the ATTCM scheme in Fig. 5. Hence, the ATTCM scheme requires approximately $24-3=21 \mathrm{dBs}$ lower transmission power compared to a fixed mode TTCM4PSK scheme, for transmitting 1 BPS from the source node to the relay nodes.

\section{CONCLUSION}

An attractive cooperative scheme was proposed based on adaptive coded modulation and distributed space-time coding for communicating over quasi-static Rayleigh fading channels. The adaptive coded modulation scheme was utilised for protecting the source-to-relay links, while the distributed space-time code was employed for enhancing the reliability of the relay-to-destination links. It was shown that mobile units equipped with a single antenna are capable establishing an energy-efficient wireless cooperative network. More specifically, the proposed cooperative ATTCM-DSTTC-2x2 scheme outperforms the uncoded non-cooperative BPSK-1x2 scheme by over $20 \mathrm{dBs}$ at a FER of $10^{-3}$, when communicating over quasi-static Rayleigh fading channels.

\section{REFERENCES}

[1] V. Tarokh, N. Seshadri, and A. R. Calderbank, "Space-time codes for high data rate wireless communication: Performance criterion and code construction," IEEE Transactions on Information Theory, vol. 44, pp. 744-765, March 1998.

[2] Y. Li, "Distributed coding for cooperative wireless networks: An overview and recent advances," IEEE Communications Magazine, vol. 47, pp. 71-77, August 2009.

[3] A. Sendonaris, E. Erkip and B. Aazhang, "User cooperation diversity part I: System description," IEEE Transactions on Communications, vol. 51(11), pp. 1927-1938, 2003.

[4] N. Laneman, D. N. C. Tse and G. W. Wornell, "Cooperative diversity in wireless networks: efficient protocols and outage behavior," IEEE Trans. on Information Theory, vol. 50, no. 12, pp. 3062-3080, 2004.

[5] E. Zimmermann, P. Herhold and G. Fettweis, "On the performance of cooperative relaying protocols in wireless networks," European Transactions on Telecommunications, vol. 16, no. 1, pp. 5-16, 2005.

[6] C. Wong and L. Hanzo, "Upper-bound performance of a wideband burstby-burst adaptive modem," IEEE Transactions on Communications, vol. 48, pp. 367-369, March 2000.

[7] A.J. Goldsmith and S. Chua, "Adaptive coded modulation for fading channels," IEEE Transactions on Communications, vol. 46, pp. 595602, May 1998.

[8] S. X. Ng, J. Y. Chung, P. Cherriman and L. Hanzo, "Burst-by-burst adaptive decision feedback equalised tcm, ttcm and bicm for h.263assisted wireless video telephony," IEEE Transactions on Circuits and Systems for Video Technology, pp. 363-374, March 2006.

[9] P. Robertson and T. Wörz, "Bandwidth-efficient turbo trellis-coded modulation using punctured component codes," IEEE Journal on Selected Areas in Communications, vol. 16, pp. 206-218, Feb 1998.

[10] G. Ungerböck, "Channel coding with multilevel/phase signals," IEEE Transactions on Information Theory, vol. IT-28, pp. 55-67, January 1982.

[11] H, Ochiai, P. Mitran and V. Tarokh, "Design and analysis of collaborative diversity protocols for wireless sensor networks," in Proceedings of IEEE VTC Fall, (Los Angeles, USA), pp. 4645 - 4649, 26-29 September 2004.
[12] L. Hanzo, T.H. Liew and B.L. Yeap, Turbo Coding, Turbo Equalisation and Space Time Coding for Transmission over Wireless channels. New York, USA: John Wiley IEEE Press, 2002. 